\title{
Challenging facial pigmented lesions: values and limits of confocal microscopy
}

\author{
Marina Agozzino ${ }^{1}$, Teresa Russo ${ }^{1}$, Marco Ardigò $^{2}$, Vincenzo Piccolo ${ }^{1}$, Massimo Mascolo $^{3}$, \\ Stefania Staibano ${ }^{3}$, Roberto Alfano ${ }^{4}$, Giuseppe Argenziano ${ }^{1}$
}

1 Dermatology Unit, University of Campania Luigi Vanvitelli, Naples, Italy

2 Department of Dermatological Clinic, San Gallicano Dermatological Institute-IRCCS, Rome, Italy

3 Department of Advanced Biomedical Sciences, Pathology Section, University of Naples Federico II, Naples, Italy

4 Department of Anesthesiology, Surgery and Emergency, University of Campania Luigi Vanvitelli, Naples, Italy

Key words: in vivo reflectance confocal microscopy, pigmented facial lesion, lentigo maligna, seborrheic keratosis

Citation: Agozzino M, Russo T, Ardigò M, Piccolo V, Mascolo M, Staibano S, Alfano R, Argenziano G. Challenging facial pigmented lesions: values and limits of confocal microscopy. Dermatol Pract Concept. 2018;8(3):188-190. DOI: https://doi.org/10.5826/dpc.0803a08 Received: January 12, 2018; Accepted: March 7, 2018; Published: July 31, 2018

Copyright: @2018 Agozzino et al. This is an open-access article distributed under the terms of the Creative Commons Attribution License, which permits unrestricted use, distribution, and reproduction in any medium, provided the original author and source are credited.

Funding: None.

Competing interests: The authors have no conflicts of interest to disclose.

All authors have contributed significantly to this publication.

Corresponding author: Teresa Russo, MD, c/o II Policlinico, Edificio 9, Primo piano, Via Pansini 5 - 80131 Naples, Italy. Email: russo. teresa87@gmail.com.

\section{Introduction}

Pigmented facial lesions (PFL) may be difficult to diagnose clinically and dermoscopically. Nowadays, reflectance confocal microscopy (RCM) offers the possibility to assess in vivo PFL, although in challenging cases histopathology remains mandatory [1,2]. Herein a series of 3 patients with ambiguous PFL are reported.

\section{Discussion}

Clinically, all lesions were flat and brownish in color (Figures $1 \mathrm{~A}-1 \mathrm{C}$ ), while dermoscopically they revealed brownblack thickened pseudonetwork, grayish color, and rhomboidal structures (Figures 1A and 1B). Only one also exhibited scattered milia-like cysts (Figure 1C).

RCM examination of the 3 lesions, performed using a Vivascope 1500 (Lucid, Rochester, NY, USA), showed elon- gated bright tubular cords with bulbous projections (lesion A, Figure 2A), keratinocyte disarray and atypical cell aggregates around adnexal structures (lesion B, Figure 2B), and dendritic cell infiltration of adnexal structures with numerous reflective atypical roundish cells at the dermoepidermal junction (lesion C, Figure 2C). Features seen in the second and third lesions were interpreted as suggestive of lentigo maligna (LM).

Histopathology performed on excisional biopsies for all 3 lesions confirmed the diagnosis of an irritated, pigmented seborrheic keratosis (SK) of lesion A (Figure 3A); a diagnosis of melanoma in situ was made in lesion B (Figure 3B); and histopathologic features of lesion $C$ were suggestive of an irritated SK and were partially in contrast with RCM findings (Figure 3D).

These examples underline how the diagnosis of flat PFL on chronically sun-damaged skin represents one of the most challenging scenarios for dermatologists treating skin cancer. In particular, facial LM and SK may show overlapping mor- 

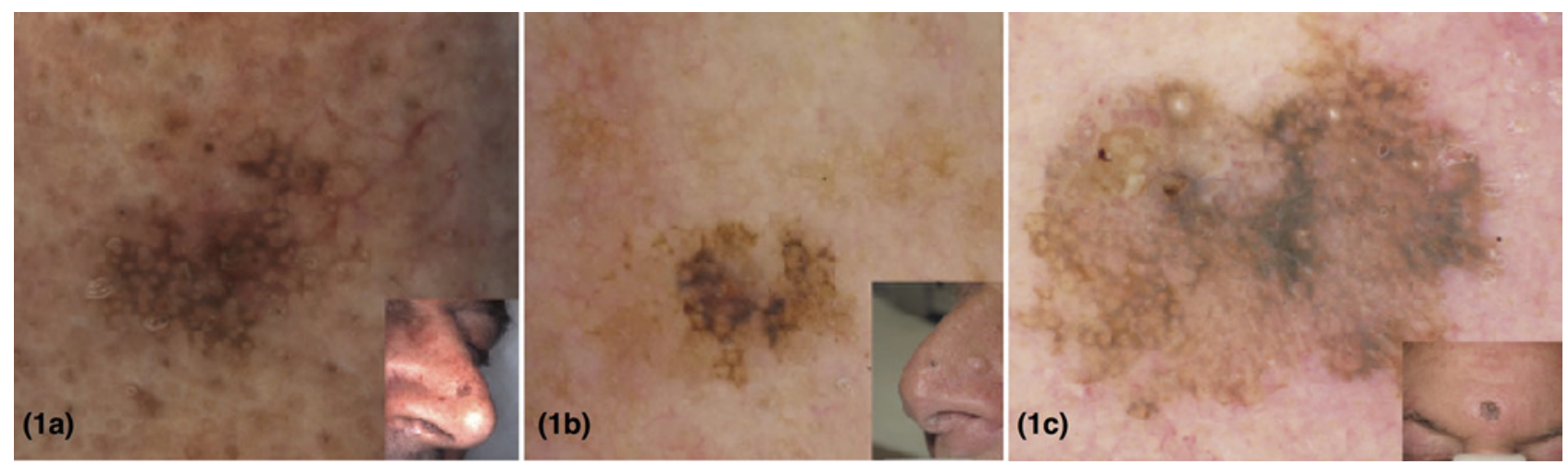

Figure 1. Dermoscopic images. (A) Lesion A and (B) lesion B show a thickened pseudonetwork and gray color in the middle area. (C) Lesion C shows sharply demarcated borders, a milia-like cyst, and looped vessels. RCM mosaics. [Copyright: (O2018 Agozzino et al.]
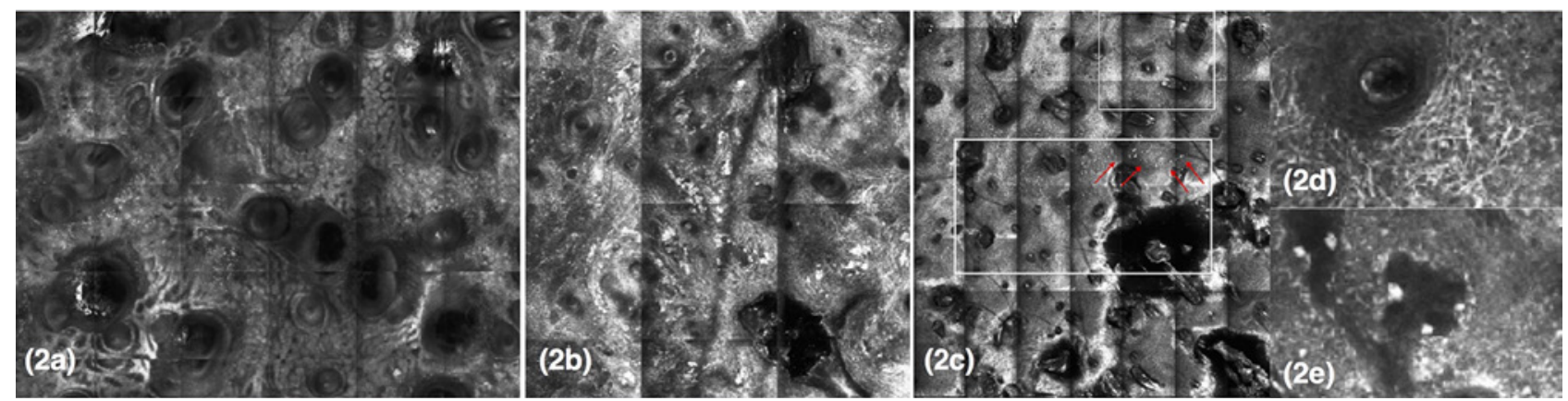

Figure 2. (A) The majority of lesion A displays elongated tubular structures (cords) and bulbous projections indicative of SK. (B) RCM images of lesion B show non-edged papillae with aggregates of atypical large nucleated cells around adnexal structures. In lesion C, the epidermis reveals $(\mathrm{C})$ a disarrayed honeycomb pattern with multiple atypical reflective cells around follicular openings (red arrows) and keratin-filled invaginations in the upper left part of the image, (D) numerous cells with dendritic processes around follicles, and (E) melanophages inside dermal papillae. [Copyright: (C2018 Agozzino et al.]
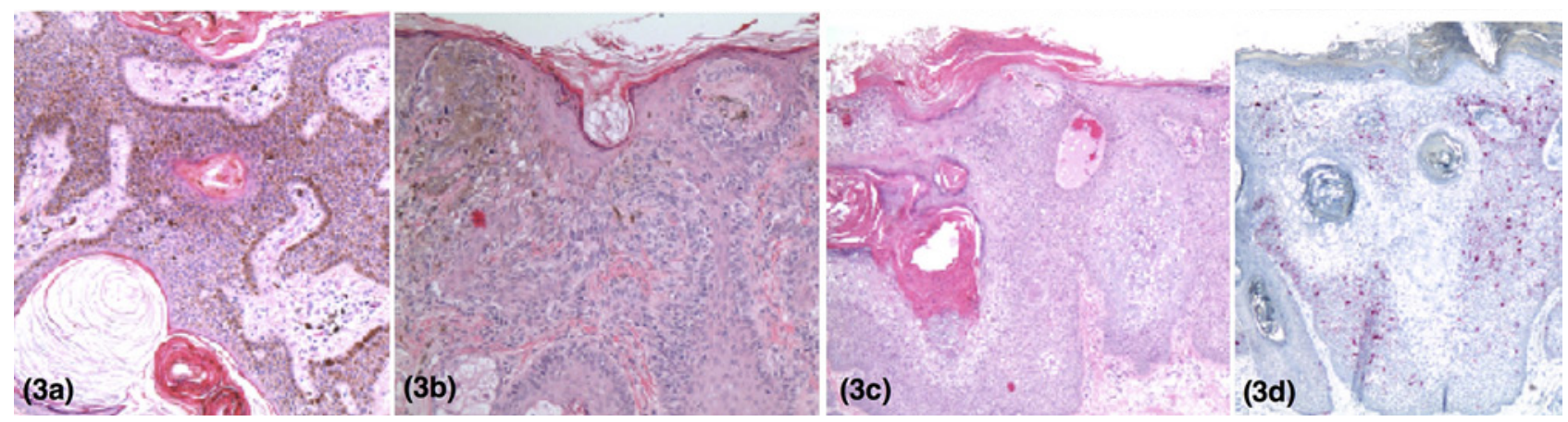

Figure 3. Histopathology. (A) Lesion A shows acanthosis, parakeratosis, and horn cysts (hematoxylin and eosin [H\&E] staining, original magnification $\times 50$ ). (B) Nested atypical melanocytes, pagetoid spread cells, and some perifollicular atypical melanocytes in lesion B (H\&E staining, original magnification $\times 50)$. (C) Lesion $\mathrm{C}$ reveals horn cysts, subepidermal chronic inflammation, and melanin deposits (H\&E staining, original magnification $\times 50$ ). (D) Staining for $\mathrm{S} 100$ protein highlights only some melanocytes (S100 protein, original magnification $\times 50$ ). [Copyright: $\odot 2018$ Agozzino et al.]

phological features. Irritated or regressive SK is particularly challenging when it shows clinical (eg, irregular borders, presence of multiple colors, asymmetry) and dermoscopic features (such as focal or diffuse pink or gray hue) that overlap with those of facial melanoma. Moreover, the presence in lesion $\mathrm{C}$ of perifollicular inflammatory cells and Langerhans cells, resembling dendritic melanocytes in RCM, represents a pos- sible RCM pitfall in the differentiation of PFL (Figure 2D). A detailed evaluation of the components of the folliculotropism in facial macules may help to improve the diagnostic accuracy of RCM findings [3]. The infiltration of the hair follicle was found to be indicative of LM/LMM compared to nonmelanocytic skin neoplasms, with an overall sensitivity of $96 \%$ and specificity of $83 \%$ [3]. A few bright dendrites in the epider- 
mis, but not infiltrating the hair follicle predominantly, have estimated specificity for pigmented actinic keratosis $(53 \%)$ [3]. Therefore, further studies of distribution and amount of cells, and the presence of bulging around the follicles, are necessary and could represent an important tool to help in differentiating PFL.

\section{Conclusions}

Nowadays, confocal examination provides increasingly useful information for the interpretation of doubtful facial lesions. However, histopathologic examination remains mandatory in special cases to avoid overtreatment.

\section{References}

1. Russo T, Piccolo V, Lallas A, et al. Dermoscopy of malignant skin tumours: what's new? Dermatology. 2017;233(1):64-73. doi: $10.1159 / 000472253$.

2. Guitera P, Pellacani G, Crotty KA, et al. The impact of in vivo reflectance confocal microscopy on the diagnostic accuracy of lentigo maligna and equivocal pigmented and nonpigmented macules of the face. J Invest Dermatol. 2010;130(8):2080-2091. doi: 10.1038/ jid.2010.84.

3. Persechino F, De Carvalho N, Ciardo S, et al. Folliculotropism in pigmented facial macules: differential diagnosis with reflectance confocal microscopy. Exp Dermatol. 2018;27(3):227-232. doi: 10.1111/exd.13487. 